\title{
The genetic analysis of family structured inbreeding depression studies
}

\author{
JK Kelly and MK Tourtellot \\ Department of Ecology \& Evolutionary Biology, University of Kansas, 1200 Sunnyside Ave, Lawrence, KS 66045-7534, USA
}

\begin{abstract}
An important question emerging from theoretical studies of mating system evolution is whether the fitness of a randomly extracted, fully inbred genotype will exceed the mean of outbred individuals. We introduce two statistics $\left(\boldsymbol{I}_{\mathbf{1}}\right.$ and $\left.\boldsymbol{I}_{2}\right)$ related to the probability of extracting a high line. $\boldsymbol{I}_{1}$ and $\boldsymbol{I}_{2}$ can be estimated from the family structured experimental designs typically used to estimate inbreeding depression (ID). Maximum likelihood procedures are developed from an
\end{abstract}

explicit genetic model. These yield parameter estimates and provide the likelihoods necessary to test hypotheses, for example, whether population-level ID is nonzero. Finally, we describe a new publicly available computer program titled 'IDG' (Inbreeding Depression Genetics) to execute these procedures.

Heredity (2006) 97, 346-354. doi:10.1038/sj.hdy.6800879; published online 9 August 2006

Keywords: Collinsia; inbreeding; inbreeding depression; mating systems; Mimulus; self-fertilization

\section{Introduction}

Inbreeding depression (ID) is the decline of fitnessrelated traits that frequently occurs with inbreeding. The magnitude of ID and its genetic basis are critical factors in the evolution of plant mating systems and also have important implications for agriculture and conservation (Darwin, 1876; Charlesworth and Charlesworth, 1987; Lynch et al, 1995; Keller and Waller, 2002). Historically, ID has been measured at the population level by comparing the mean phenotypes of inbred and outbred individuals. More recently, interest has focused on ID at the level of individual families. ID is estimated from the difference in mean fitness between inbred and outbred individuals within the same family, oftentimes the progeny of a single maternal plant. Variation in ID among families has been demonstrated within a variety of natural plant populations (eg, Agren and Schemske, 1993; Carr et al, 1997; Mutikainen and Delph, 1998; Chang and Rausher, 1999; Vogler et al, 1999; Takebayashi and Delph, 2000; Fishman, 2001; Rao et al, 2002; Stone and Motten, 2002).

Why is family-level ID interesting? Some theoretical studies suggest that it may be a more important determinant of mating system evolution than population-level ID (Campbell, 1986; Holsinger, 1988; Uyenoyama et al, 1993; but also see Charlesworth et al, 1990; Shultz and Willis, 1995). Consider a plant population that is self-compatible but predominantly outcrossing. A mutation occurs that induces complete self-fertilization. If this mutation escapes immediate loss, it will become fixed within an 'inbred lineage', a genotype that is fully homozygous for alleles residing in the ancestral outbred genotype. This genotype is essentially self-perpetuating

Correspondence: JK Kelly, Department of Ecology \& Evolutionary Biology, University of Kansas, 1200 Sunnyside Ave, Lawrence, KS 66045-7534, USA. E-mail: jkk@ku.edu

Received 26 December 2005; accepted 23 June 2006; published online 9 August 2006 in that selfed progeny are genetically identical (or nearly so) to their parents. If the constellation of alleles fixed within the lineage is favorable, it may displace the background population of sexual genotypes (Lande and Schemske, 1985). Of course, the spread of the selfing genotype is greatly facilitated if it can also distribute pollen to other plants while self-fertilizing its own ovules thus exploiting the 'cost of sex' (Maynard Smith, 1978).

The preceding description, in which a novel mutation induces complete selfing, is idealized. Genetic modifiers that cause incremental increases in selfing rate will not become isolated within self-perpetuating lineages and the evolutionary dynamics are a great deal more complicated (see Uyenoyama and Waller, 1991; Uyenoyama et al, 1993). However, the example does illustrate why interest has at least partially shifted from population to family level. It also points to a conceptual difficulty with measuring ID as a difference between inbred and outbred individuals within the same family. Over the long run, a selfing lineage will be competing with the entire background population and not just the outbred progeny of that family. Low or even negative family-level ID can result because the mean fitness of outbred progeny from that family is unusually low. This would not necessarily bode well for a selfing mutation that happens to occur within that family.

From an empirical point of view, we would like to determine the probability that a randomly extracted, fully inbred genotype will have a mean fitness that exceeds the mean of outbred genotypes within the population. Here, 'random extraction' implies that alleles fix randomly within a lineage over successive generations of selfing and that the lineage is founded by a random outbred genotype. In fact, populations of inbred lines have been extracted from several model species and measurements from these populations can directly estimate this probability (Simmons and Crow, 1977; Takano et al, 1987; Hughes, 1997; Willis, 1999b). As we discuss in greater detail below, most ID studies contain 
data sufficient for at least an indirect appraisal. The generation of high performing inbred lines is relevant not only to mating system evolution, but also has direct bearing on agriculture (Crow, 1987).

We introduce two statistics $\left(I_{1}\right.$ and $\left.I_{2}\right)$ related to the probability that a random inbred line will exceed the outbred mean phenotype in the population. Neither $\boldsymbol{I}_{\mathbf{1}}$ nor $I_{2}$ yields the probability directly, except under specific assumptions about the fitness distribution across inbred lines. However, each should be positively correlated with this probability and thus represents a useful abstraction of the genetic information resident within ID experiments. In the second section of the paper, $\boldsymbol{I}_{\mathbf{1}}$ and $\boldsymbol{I}_{\mathbf{2}}$ are directly estimated from the observed distribution of inbred line means within a large experimental study of Mimulus guttatus (Kelly, 2005a). Estimates from multiple traits support our contention that $\boldsymbol{I}_{\mathbf{1}}$ and $I_{2}$ are correlated with the probability that a line will exceed the outbred mean. The third part of the paper develops methods for estimating $\boldsymbol{I}_{1}$ and $\boldsymbol{I}_{2}$ from three standard experimental designs. The estimation procedures are based on an explicit genetic model and provide a likelihood ratio test for the presence of population-level ID. Finally, we describe a new computer program titled 'IDG' (Inbreeding Depression Genetics) to execute these procedures.

\section{ID statistics}

Let $M$ denote the outbred mean for the trait under consideration. The mean value of fully inbred genotypes is defined to be $M-\beta$, where $\beta$ is the 'inbreeding load'. As inbreeding typically depresses trait values, we expect that $\beta$ should usually be positive. Finally, let $V_{\mathrm{GI}}$ denote the (genetic) variance among fully inbred lines. Given these definitions, we will consider the following two aggregate statistics:

$$
\mathbf{I}_{\mathbf{1}}=\frac{(M-\beta)^{2}+V_{\mathrm{GI}}}{M^{2}}
$$

and

$$
\boldsymbol{I}_{2}=\frac{\sqrt{V_{\mathrm{GI}}}}{\beta}
$$

Higher values for $\boldsymbol{I}_{\mathbf{1}}$ and $\boldsymbol{I}_{\mathbf{2}}$ imply an increased probability that if an outbred genotype produces a fully inbred descendant, its phenotypic value (fitness) exceed the outbred mean.

The first statistic $\left(\boldsymbol{I}_{\mathbf{1}}\right)$ is derived from Chebyshev's inequality, an identity concerned with the probability of extreme outcomes (Feller, 1968, p 233). Let the random variable $X$ signify the fitness of a randomly extracted inbred line. Chebyshev's inequality implies:

$$
\operatorname{Prob}[X \geq M] \leq \frac{\mathrm{E}\left[X^{2}\right]}{M^{2}}=\frac{(\mathrm{E}[X])^{2}+\operatorname{Var}[X]}{M^{2}}
$$

assuming that $X$ is non-negative. Noting that $\mathrm{E}[X]=M-\beta$ and $\operatorname{Var}[X]=V_{\mathrm{GI}}$, the right-hand side (RHS) of Equation (3) becomes equal to $I_{1}$.

Chebyshev's inequality only places an upper bound on the probability that a line exceeds the outbred mean. In fact, $\boldsymbol{I}_{\mathbf{1}}$ may often exceed 1 . The actual probability depends on the shape of the distribution for $X$. If the distribution is normal, the second statistic $\left(I_{2}\right)$ provides the information necessary to predict probabilities. The fraction of density in the 'tail' of the normal distribution is a simple function of the standard deviation (here, the tail is the collection of inbred lines that exceed the outbred mean). Referring to tabulations of the standard normal (eg, Rice, 1989, p 558), we find that the probability that a random line exceeds $M$ is only 0.023 if $I_{2}=\frac{1}{2}$. This probability increases to 0.159 if $I_{2}=1$ and to 0.309 if $\boldsymbol{I}_{\mathbf{2}}=2$.

$I_{1}$ and $I_{2}$ are dimensionless ratios, a feature that facilitates comparisons across different traits and studies. However, there are important statistical issues associated with ratios of estimators (Rice, 1989, pp 146-147). In particular, substantial bias is introduced if the denominator has a large standard error (SE). This is unlikely to be a difficulty for $\boldsymbol{I}_{\mathbf{1}}$, because in all but the smallest experiments, the SE of $M$ should be small relative to its estimated value. However, this need not be true for estimates of $\beta$. Estimates for $\boldsymbol{I}_{2}$ are ruled 'suspect' by the IDG program if the SE of $\beta$ is greater than $\frac{1}{4}$ its estimated magnitude.

$I_{1}$ requires that trait values are positive which should usually be true of measurement on their original scale. However, log-transformation of fractional values, for example, proportion surviving, will yield negative values. This can be remedied simply by adding a constant to all measurements before calculating $\boldsymbol{I}_{\mathbf{1}}$. A second issue concerns the logical derivation of $\boldsymbol{I}_{\mathbf{1}}$. We assumed that inbreeding decreases trait values and that high trait values are favorable. However, for characters such as time to sexual maturity, inbreeding may increase trait values and lower values may be favorable. For such situations, $\boldsymbol{I}_{\mathbf{1}}$ can be adapted to predict the emergence of low lines simply by reversing the sign of $\beta$ before substituting it into Equation (1), thus insuring that $(M-\beta)^{2}<M^{2}$.

\section{A direct study of $I_{1}$ and $I_{2}$ in $M$. guttatus}

A large collection of inbred lines have been extracted from a single natural population of M. guttatus (Willis, 1999a; Kelly and Arathi, 2003). Each line was initiated from a single outbred genotype and synthesized by successive generations of single seed descent (selffertilization with random selection of progeny). These lines each had between seven and nine generations of selfing in their ancestry and line inbreeding coefficients greater than 0.99 (high homozygosity confirmed with genetic markers: Willis, 1999a; Liza Holeski, unpublished results). As part of a larger breeding design, the lines were randomly paired and crossed to produce F1 families. Each line was also self-fertilized to produce a large collection of genetically identical seeds. Progeny of both types were grown simultaneously and each plant was measured for a number of morphological traits and male fitness components. A detailed description of the crossing and measurement procedures is given elsewhere (Kelly, 2005a).

Figure 1 illustrates the distribution of inbred line means for corolla width, the number of pollen grains per flower, and the estimated number of viable pollen grains per flower. The outbred mean, $M$, is superimposed upon these distributions. Estimates for $M, \beta$, and $V_{\mathrm{GI}}$ were obtained by applying one-way ANOVAs to either the collection of F1s (for $M$ ) or inbred line progeny (for $\beta$ and $\left.V_{\mathrm{GI}}\right)$. Families, either F1 or line, define the groups in 
348
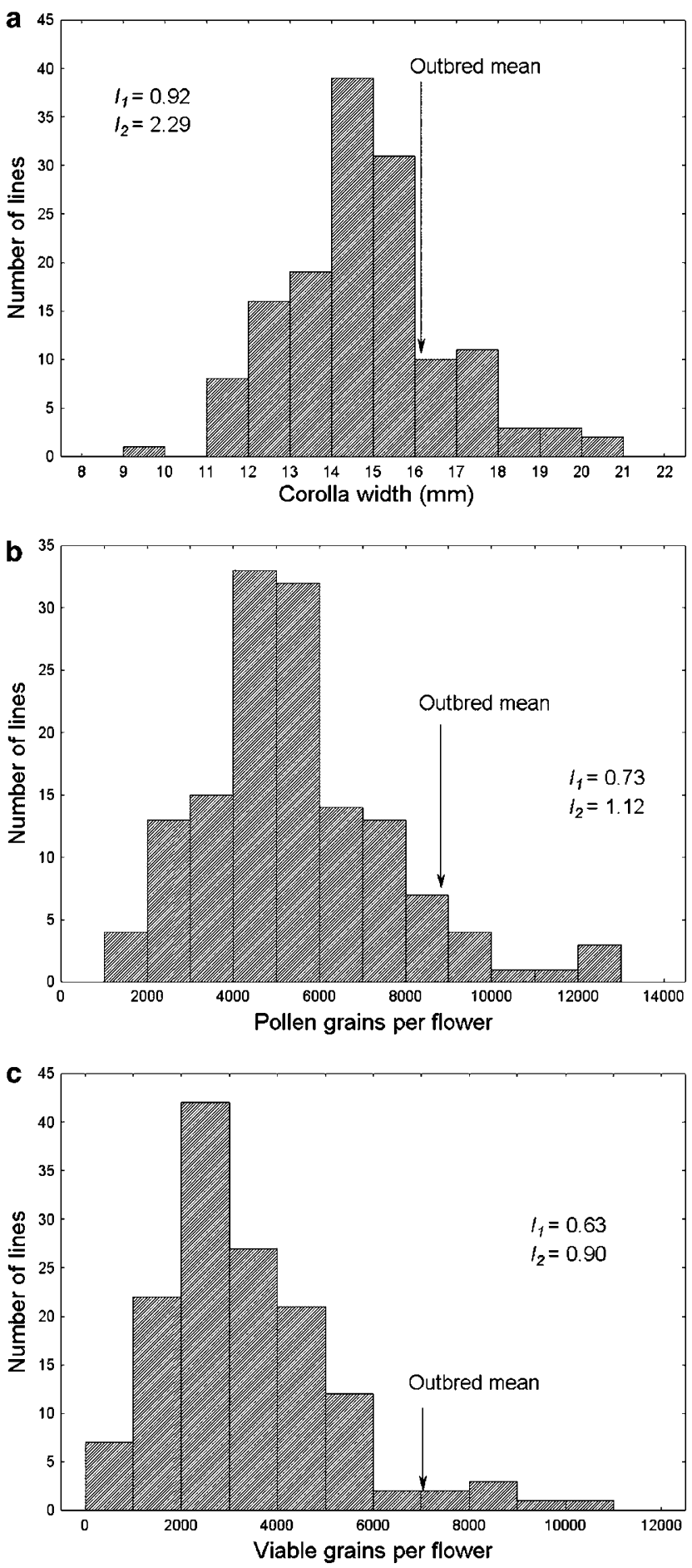

Figure 1 The distributions of inbred line means are given for (a) corolla width, (b) total pollen per flower, and (c) viable pollen per flower.

these ANOVAs. Using F1 families, the ANOVA grand mean $(\mu)$ estimates $M$. With the lines, $\mu$ estimates $(M-\beta)$ and the among-group variance estimates $V_{\mathrm{GI}}$. The values for $I_{1}$ and $I_{2}$ were obtained by substituting the estimates for $M, \beta$, and $V_{\mathrm{GI}}$ into Equations (1-2).

$I_{1}$ and $I_{2}$ correlate positively with the observed proportion of inbred lines that exceed the outbred mean (Figure 1). Corolla width yields the highest values for $\boldsymbol{I}_{\mathbf{1}}$ and $\boldsymbol{I}_{2}$, and for this trait, 27 of 143 inbred line means exceed $M$. For total pollen per flower, $I_{1}$ and $I_{2}$ are substantially reduced and only nine inbred lines exceed $M$. The lowest values for $\boldsymbol{I}_{\mathbf{1}}$ and $\boldsymbol{I}_{\mathbf{2}}$ were obtained for the estimated number of viable pollen grains per flower, where only seven lines had means greater than $M$. As line means are estimated with error, these numbers likely overestimate the proportion of lines that exceed the outbred mean. If we include only lines that exceed $M$ by two SEs, the number of high lines is reduced to 12 for corolla width, two for pollen number, and three for viable pollen per flower.

\section{Estimation of $\boldsymbol{I}_{\mathbf{1}}$ and $\boldsymbol{I}_{\mathbf{2}}$ from standard designs}

For the data of Figure 1, $\boldsymbol{I}_{\mathbf{1}}$ and $\boldsymbol{I}_{\mathbf{2}}$ are superfluous because one can directly estimate the probability of extracting a high performing inbred line. However, most ID studies do not involve a large collection of fully inbred lines. Oftentimes, individuals are only partially inbred, for example, the selfed progeny of outbred parents. However, because most ID experiments are breeding designs (see Figure 2), $\boldsymbol{I}_{\mathbf{1}}$ and $\boldsymbol{I}_{\mathbf{2}}$ can still be estimated by applying a quantitative genetic model to the data. In a randomly mating population, the genetic variance is typically parsed into two components, the additive variance, $V_{\mathrm{a}}$, and dominance variance, $V_{\mathrm{d}}$ (Lynch and Walsh, 1998, Ch 4-7; Falconer and Mackay, 1996, Ch 8-9). With inbreeding, the genetic variance depends not only on $V_{\mathrm{a}}$ and $V_{\mathrm{d}}$, but also on several 'inbreeding components' (Harris, 1964; Cockerham, 1983; Shaw et al, 1998): the covariance of additive and dominance effects (denoted $C_{a d}$ ), the inbreeding dominance variance $\left(V_{\mathrm{di}}\right)$, and the sum of squared ID at individual loci $\left(H^{*}\right)$. If there are only two alleles per locus, the number of terms is reduced by one because $H^{*}=V_{\mathrm{d}}$ (Cockerham and Weir, 1984). These components determine not only the genetic variance, but also the covariances among relatives. In the analyses described below, we assume that quantitative trait loci (QTL) are in linkage equilibria, at least in the experimental population if not the natural population.

$I_{1}$ and $I_{2}$ require estimates for $M, \beta$, and $V_{\mathrm{GI}}$. The most challenging is $V_{\mathrm{GI}}$, which is equal to $2 V_{\mathrm{a}}+4 C_{\mathrm{ad}}+V_{\mathrm{di}}$ (Cockerham and Weir, 1984). However, some experimental designs allow us to estimate $V_{\mathrm{GI}}$ without estimating the individual components. Estimation of $M$ and $\beta$ is straightforward given the sample means for both inbred and outbred plants, although it is critical that $f$ is known for the inbred individuals. As $\beta$ represents the difference in mean phenotype between outbred and fully inbred $(f)$ plants, the observed difference must be multiplied by a factor if inbred individuals have $f<1$. Most frequently $f=0.5$, in which case, $\beta$ is estimated as twice the difference between inbred and outbred samples.

The crossing scheme for three experimental designs is depicted in Figure 2. Design 1, in which each family in centered on a single maternal plant (Figure 2a), is most commonly used. We assume that maternal plants are randomly selected from the background population and all are either outbred or fully inbred $(f=1)$. Each maternal plant is self-fertilized to produce one set of progeny (denoted Inbred subfamily in Figure 2a) and receive pollen from one or more sires to produce an additional set of progeny (denoted oubred subfamily). Progeny from both types of subfamily are grown 

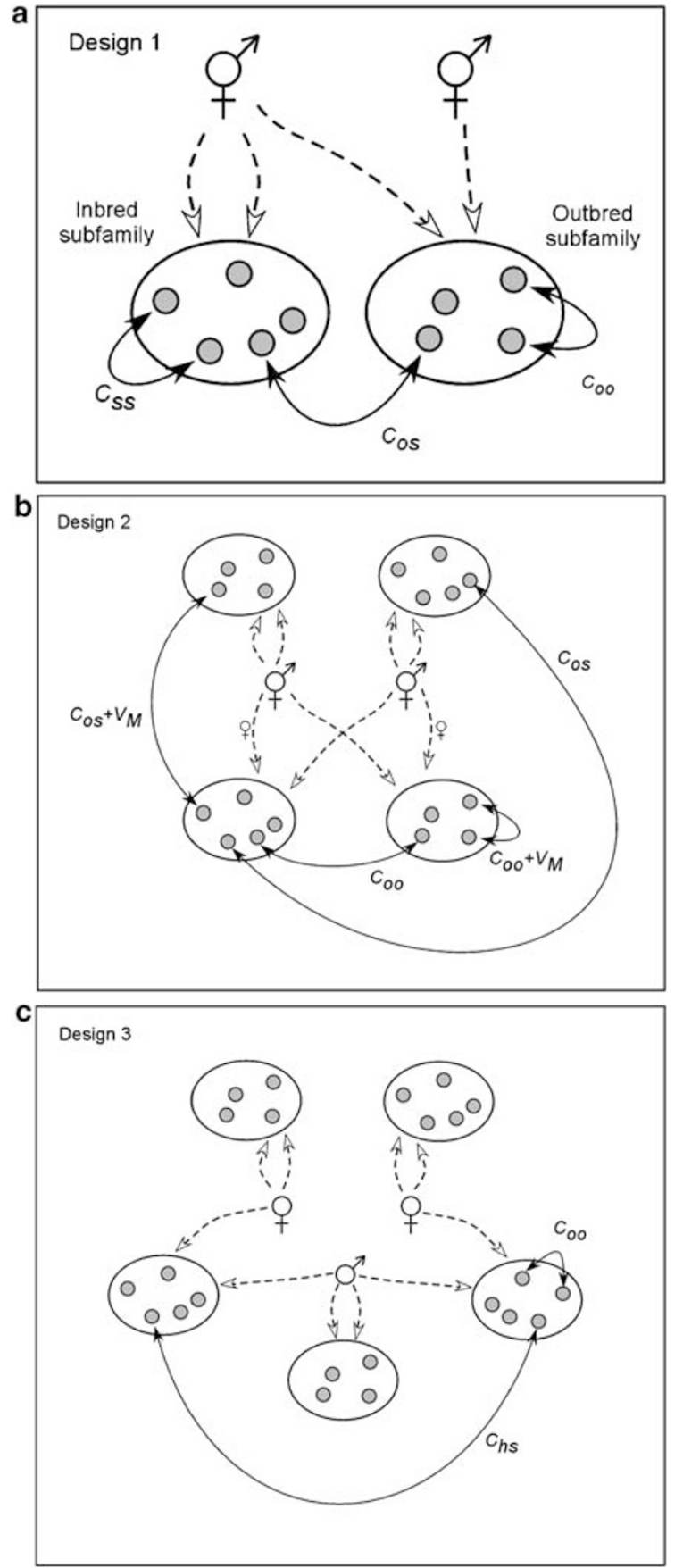

Figure 2 A diagram of the crosses and self-fertilizations design used in (a) Design 1, (b) Design 2, and (c) Design 3. Small filled circles denote individual plants. Double-headed arrows denote comparisons that define observational variance components. The arrows with broken lines represent transmission of a gamete.

simultaneously and measured for a range of traits related to fitness (eg, germination, survival, number of flowers produced, pollen produced per flower, ovules per flower). The full set of progeny from each pair of parental plants (both outbred and inbred) constitutes a family in this design.

There are three different comparisons among relatives: $\mathrm{C}_{\mathrm{OO}}$ is the covariance between individuals within outbred subfamilies, $C_{S S}$ is the covariance between individuals within inbred subfamilies, and $C_{O S}$ is the covariance between outbred and inbred individuals within the same family. In this case, the covariances within groups are equivalent to variances among group means: $C_{\mathrm{OO}}$ is equal to the variance among the true means of outbred subfamilies and $C_{S S}$ is equal to the variance among true means of inbred subfamilies. For the genetic analysis of this design, we distinguish four cases (the factorial combinations of two factors): maternal plants are either outbred (eg, Koelewijn et al, 1999) or inbred (eg, Agren and Schemske, 1993), and there is either a single sire per dam (eg, Kalisz, 1989) or that many (unrelated) sires contribute to the pollen mixture transferred to each dam (eg, Fishman, 2001). Let $B$ denote the observed difference in mean phenotype between inbred and outbred plants. If the maternal parents are outbred, then $\beta=-2 B$. If maternal parents are fully inbred, then $\beta=-B$.

An important limitation of Design 1 is that all individuals are related through a single maternal plant. As a consequence, variances and covariances are potentially contaminated by maternal effects. To extract genetic variance components, and thus estimate $\boldsymbol{I}_{\mathbf{1}}$ and $\boldsymbol{I}_{\mathbf{2}}$ we must assume that maternal effects are absent, an assumption not necessary for Designs 2 and 3. Using the parameterization of Cockerham and Weir, 1984 (see Appendix), the predicted genetic variance among fully inbred lines can be calculated from the observed covariances:

\section{Parents outbred}

Single sire per dam:

$$
V_{\mathrm{GI}}=2\left(C_{\mathrm{OO}}+V_{\mathrm{I}}-V_{\mathrm{O}}\right)
$$

Many sires per dam:

$$
V_{\mathrm{GI}}=8 / 5\left(3 C_{\mathrm{OO}}-2 C_{\mathrm{OS}}+C_{\mathrm{SS}}+V_{\mathrm{I}}-V_{\mathrm{O}}\right)
$$

\section{Parents fully inbred}

Single sire per dam:

$$
V_{\mathrm{GI}}=1 / 4\left(C_{\mathrm{OO}}+3 C_{\mathrm{SS}}+V_{\mathrm{I}}-V_{\mathrm{O}}\right)
$$

Many sires per dam: $V_{\mathrm{GI}}=C_{\mathrm{SS}}$

where $V_{\mathrm{O}}$ is the variance among outbred plants and $V_{\mathrm{I}}$ is the variance among inbred plants. In all cases, $V_{\mathrm{GI}}=0$ if RHS is negative.

Estimation of $\boldsymbol{I}_{\mathbf{1}}$ and $\boldsymbol{I}_{\mathbf{2}}$ from Design 1 is illustrated by reanalysis of data from a study of Collinsia verna (Kalisz, 1989). Table 1 summarizes the output of the IDG programs (described below) for three characters, seed mass, day of germination, fecundity. Each character is analyzed on the original scale of measurement and after log-transformation. Consistent with the analyses of Kalisz (1989), IDG indicates significant population-level ID for each trait $(\beta>0) . I_{1}$ and $I_{2}$ are greatest for seed mass, intermediate for days, and lowest for fecundity. Transformation generally increases $\boldsymbol{I}_{\mathbf{1}}$ and $\boldsymbol{I}_{\mathbf{2}}$, with the pronounced effect on $\boldsymbol{I}_{1}$ for fecundity. Most $\boldsymbol{I}_{2}$ values are suspect due to the relatively large SEs associated with $\beta$ estimates.

The IDG program uses maximum likelihood to estimate the genetic parameters for Designs 1, 2, and 3 of Figure 2. The fitted model(s) are contingent on the design and thus so are the estimated parameters. In all 
Table 1 Parameter estimates (and SE) from the reanalysis of data from Kalisz (1989)

\begin{tabular}{|c|c|c|c|c|c|}
\hline Trait & M & $\beta$ & $\mathrm{V}_{G I}$ & $\mathbf{I}_{1}$ & $\mathbf{I}_{2}$ \\
\hline Seed mass & $340.4(7.1)$ & $36.2(15.9)$ & $5045(2034)$ & 0.84 & $1.96^{\mathrm{a}}$ \\
\hline Day of germination & $9.92(0.60)$ & $2.70(1.22)$ & $4.02(12.73)$ & 0.57 & $0.74^{\mathrm{a}}$ \\
\hline Fecundity & $45.3(2.3)$ & $22.6(5.1)$ & $0.0(249)$ & 0.25 & 0.00 \\
\hline \multicolumn{6}{|c|}{ Log-transformed (base 10) values } \\
\hline Seed mass & $2.52(0.01)$ & $0.056(0.022)$ & $0.0135(0.0036)$ & 0.96 & $2.09^{\mathrm{a}}$ \\
\hline Day of germination & $0.86(0.03)$ & $0.18(0.06)$ & $0.00(0.02)$ & 0.62 & $0.00^{\mathrm{a}}$ \\
\hline Fecundity & $1.597(0.016)$ & $0.235(0.047)$ & $0.0(0.016)$ & 0.73 & 0.00 \\
\hline
\end{tabular}

${ }^{a}$ Estimate for $I_{2}$ is suspect due to estimation error (high SE) associated with $\beta$.

Table 2 Estimates (and SE) for $V_{\mathrm{GI}}, M$, and $\beta$ from the re-analysis of data from Kelly and Arathi (2003) and Kelly (2003)

\begin{tabular}{lccccc}
\hline Trait & $\mathrm{M}$ & $\beta$ & $\mathrm{V}_{a}+\mathrm{V}_{d}$ & $V_{G I}$ & $\mathbf{I}_{1}$ \\
\hline Corolla width & $19.30(0.16)$ & $1.86(0.16)$ & 1.91 & $3.38(0.62)$ & 0.83 \\
Total pollen per flower & $12250(214)$ & $5382(256)$ & 4041000 & $5940000(30890)$ & 0.35 \\
Viable pollen per flower & $10220(232)$ & $5551(257)$ & 5500000 & $5588000(124800)$ & 0.26 \\
& & & & & \\
Log-transformed fitness measures & & & & & \\
Ln (pollen per flower) & $9.31(0.02)$ & $0.73(0.04)$ & 0.019 & $0.19(0.03)$ & 0.85 \\
Ln (viable pollen) & $9.06(0.03)$ & $1.07(0.05)$ & 0.038 & $0.44(0.06)$ & 0.60 \\
\hline
\end{tabular}

Natural log-transformed values for the pollen traits are also analyzed. The estimate genetic variance among outbred genotypes $\left(V_{\mathrm{a}}+V_{\mathrm{d}}\right)$ is reported.

cases, however, the likelihood analysis yields SEs for parameter estimates from the asymptotic dispersion matrix. The likelihood values from different models also provide a means of hypothesis testing using likelihood ratios. For all designs, one can test for population-level ID, that is, whether $\beta=0$. Tests regarding the variance components are specific to each design. These programs are written in VBA (Visual Basic for Applications) and run within the Microsoft Excel environment. The 'AddIn' can be downloaded from http:/ / www.ku.edu/ eeb/ faculty/kellyj.html. There is also a sample data set for each design and a detailed description of the relevant studies.

In Design 2 (Figure $2 \mathrm{~b}$ ), each family is a small diallel synthesized by reciprocally crossing and selfing two parents (eg, Willis, 1993). Each family contains four subfamilies, two selfed progeny sets and two oubred progeny sets. The same genetic relationships exist within Design 2 as are present in Design 1, that is, $C_{O O}, C_{O S}$, and $C_{\mathrm{SS}}$. However, because crosses are performed reciprocally, we do not need to assume that maternal effects are absent. We can parse both $C_{\mathrm{OO}}$ and $C_{\mathrm{O}}$ according to whether individuals share the same maternal parent or not. The variance of maternal effects, $V_{\mathrm{M}}$, contributes to covariances among individuals that are related through a maternal parent but not to relationships limited to a common male parent. As with Design 1, we must distinguish cases where the parents are outbred $(\beta=-2 B)$ and the parents are fully inbred $(\beta=-B)$. We can use Equation (4a) to estimate $V_{\mathrm{GI}}$ when parents are outbred and Equation (4c) when parents are fully inbred. However, the variances and covariances are discounted for any contribution of maternal effects (Figure 2b). In fact, an analysis of the sample data set that accompanies the program, Willis' study of $M$. guttatus (Willis, 1993), clearly demonstrates a significant contribution of maternal effects to the genetic variance in fecundity.
Design 3 is a direct extension of the standard full-sib/ half-sib scheme routinely used to estimate heritability (Figure 2c). A series of sires is each mated to a distinct set of unrelated dams. Each parent (sires and dams) is also self-fertilized (eg, Kelly and Arathi, 2003). Here, we distinguish two different comparisons among oubred siblings, the covariance among full sibs $\left(C_{\mathrm{OO}}\right)$, and the covariance among half-sibs $\left(C_{\mathrm{HS}}\right)$. For this design, it is most efficient to directly estimate the causal components, $V_{\mathrm{a}}, V_{\mathrm{d}}, C_{\mathrm{ad}}$, and $V_{\mathrm{di}}$, because they are fewer than the number of observational comparisons within the design (Shaw et al, 1998).

Design 3 is illustrated by an application to another study of M. guttatus. Kelly and Arathi (2003) used fully inbred parents in a breeding design with two dams per sire. The progeny were grown to maturity in the greenhouse and measured for flower morphology, development rate and male fitness components. Genetic parameter estimates for corolla width, total pollen per flower, and viable pollen per flower are given in Table 2. Log-tranformed values for the pollen traits were also analyzed. Likelihood ratio tests confirm that all traits exhibit significant population-level ID. Tests for maternal effects were not significant. Estimates of $\boldsymbol{I}_{\mathbf{1}}$ and $\boldsymbol{I}_{\mathbf{2}}$ from the Design 3 study of M. guttatus (Table 2) are substantially lower than the comparable values given in Figure 1 for corolla width, total pollen per flower, and viable pollen per flower. This is notable given that the two experiments use parents extracted from the same natural population. A critical difference is that the plants of Table 2 were grown in a greenhouse with a mixture of natural and artificial light, while those used for Figure 1 matured in a growth chamber. Plants develop more rapidly in the growth chamber (by an average of about 10 days) and produce their first flower at a smaller size. This comparison of studies suggests that the difference between inbred and outbred plants becomes proportionately larger with development (aging). 


\section{Discussion}

Genetic purging is often invoked in arguments about the evolution of mating systems. Purging is the effect of selection on deleterious mutations, and in particular, the accelerated elimination of such mutations as inbreeding reveals them in homozygotes (Barrett and Charlesworth, 1991; Byers and Waller, 1999). Purging can occur at the population level wherein the genetic load (measured as population-level ID or $\beta$ ) is reduced by generations of increased inbreeding. However, it has also been hypothesized to occur at the scale of individual families or 'selfing lineages.' A genetic modifier that increases the rate of selfing might effectively purge deleterious mutations from its lineage, yielding descendants that are relatively low in genetic load (Uyenoyama et al, 1993, review much of the relevant theory).

It is critical to recognize that selection is only one source of associations between mating system modifiers and the background genotype that determines fitness. All new mutations occur in a novel background. If a mutation induces selfing and thus initiates an inbreeding lineage, the background genotype will subsequently change due to Mendelian segregation, that is, random fixation of alleles at loci that were heterozygous in the initial genotype. Purging within lineages makes this fixation process nonrandom to some degree. However, the simulation study of Shultz and Willis (1995) suggests that stochastic and/or segregation effects have a much greater impact on variation in ID among families than does lineage-specific purging. The same conclusion may often apply when considering associations between mating system modifiers and fitness determining alleles at other loci.

The two statistics developed here, $\boldsymbol{I}_{\mathbf{1}}$ and $\boldsymbol{I}_{\mathbf{2}}$, essentially measure the effects of variation in initial background and subsequent segregation within selfing lineages. These processes determine $V_{\mathrm{GI}}$, the variance among fully homozygous genotypes. $\boldsymbol{I}_{\mathbf{1}}$ and $\boldsymbol{I}_{\mathbf{2}}$ are simple functions of $V_{\mathrm{GI}}$ and each quantity should be positively related to the probability that a randomly extracted inbred line will exceed the mean phenotypic value (fitness) of outbred genotypes in a population. Neither quantity yields the probability directly except under special circumstances. However, some guidance for interpreting values of $\boldsymbol{I}_{\mathbf{1}}$ and $I_{2}$ is provided by Figure 1 . A randomly extracted line is quite unlikely to exceed $M$ if $I_{1}<0.5$ or $I_{2}<1$. A substantial fraction of lines may exceed the outbred mean if $\boldsymbol{I}_{1}>1$ or $\boldsymbol{I}_{2}>2$. Of course, $\boldsymbol{I}_{1}$ and $\boldsymbol{I}_{\mathbf{2}}$ will likely be informative only with reasonably accurate estimates for $M, \beta$, and $V_{\mathrm{GI}}$. In our reanalysis of the Collinsia study (Kalisz, 1989), $\boldsymbol{I}_{2}$ estimates for 'seed mass' and 'days to germination' are deemed suspect because the SE associated with $\beta$ is relatively high (Table 1 ).

The quantitative relationship between the high line probability and our statistics $\left(\boldsymbol{I}_{1}\right.$ and $\left.\boldsymbol{I}_{2}\right)$ depends on the form of the distribution of phenotypic (fitness) values. Comparisons of $\boldsymbol{I}_{\mathbf{1}}$ or $\boldsymbol{I}_{\mathbf{2}}$ across traits or taxa are thus most meaningful if the relevant distributions are similar in character. The distribution is also important for the adequacy of the underlying quantitative genetic model, which assumes that genetic loci combine additively to determine phenotype. Scale transformations are often applied (the logarithm, square-root, or arcsin squareroot) to make data more amenable to analysis. For our purposes, Wright (1952) outlined the most relevant criteria for scale transformation: 'The best scale for the purpose of analysis is one on which the effects of factors (genetic and environmental) are as nearly additive as possible.' For example, Wright's criteria suggest a log-transformation of each trait in the Kalisz (1989) data set (Table 1) because the original distributions were right-skewed while log-transformation yields approximate normality within both outbred and inbred samples.

\section{Applications}

The IDG program is devised to facilitate the application of quantitative genetic models to studies of ID. The 'fitness components' measured in ID studies are generally quantitative traits. While analysis of variance (ANOVA) is typically used for data analysis (Johnston and Schoen, 1994), the quantitative genetic perspective can provide a more accurate description of variation. For example, even the simplest genetic models (Equations $(4 a-d))$, predict that the variance among inbred families will differ from the variance among outbred families within an ID breeding design. These two variances are constrained to be equal in the standard ANOVA (Kelly, 2005b; see also Fox, 2005; Moorad and Wade, 2005). Both generic statistical and quantitative genetic models impose simplifying assumptions, but these assumptions become explicit when data are analyzed within a quantitative genetic framework. Finally, the motivation for most ID studies of natural populations is to address evolutionary questions. It is sensible to test predictions derived from genetic models (eg, Charlesworth et al, 1990; Uyenoyama and Waller, 1991) by applying explicitly genetic models to the data.

The reanalysis of the sample data sets illustrates how nontrivial results can emerge from a quantitative genetic treatment data (Tables 1 and 2). Contrast the variance component estimates for 'days to germination' in C. verna with pollen traits of $M$. guttatus (each trait log-transformed). For germination in C. verna, the estimated outbred genetic variance is greater than the inbred genetic variance $\left(V_{\mathrm{O}}>V_{\mathrm{I}}\right.$ and $\left.C_{\mathrm{OO}}>C_{\mathrm{SS}}\right)$ while the opposite obtains for the Mimulus pollen traits $\left(V_{\mathrm{GI}} \gg\right.$ $\left.V_{\mathrm{a}}+V_{\mathrm{d}}\right)$. This difference is notable when considering the genetic basis for ID. If fitness variation is due to deleterious mutations that are rare and partially recessive, then inbreeding should substantially inflate the genetic variance (Robertson, 1952; Kelly, 1999; Charlesworth and Hughes, 2000).

There are several possible explanations for why $V_{\mathrm{O}}$ might be greater than $V_{\mathrm{I}}$ for the Collinsia data. First, rare (partially) recessive alleles may be only one component of genetic variation, even if they are the primary cause of ID in the trait. Second, epistasis of a simple scalar kind can inflate $V_{\mathrm{O}}$ relative to $V_{\mathrm{I}}$. If the variance among genotypes at a particular locus increases with the mean of the genetic background, $V_{\mathrm{O}}$ can exceed $V_{\mathrm{I}}$ when the overall mean of outbred plants is substantially higher than that of inbred plants $(\beta \gg 0)$. This is not the most likely explanation for $V_{\mathrm{O}}>V_{\mathrm{I}}$ of 'days to germination' in $C$. verna because the inbred plants have a higher mean but lower variance than outbred plants.

\section{Comparisons among traits and species}

The parameters of quantitative genetic models are directly comparable across studies. Differences in experi- 
mental design, for example, the type of parents used for crosses (inbred or outbred), have hindered comparisons among ID studies. Consider the standard measure of ID at the population level: $\delta$ is calculated as 1 minus the ratio of the mean fitness of inbred individuals to the mean fitness of outbred individuals. This ratio depends not only on the genetic architecture of the trait but also on the difference in $f$ between inbred and outbred individuals. Outbred progeny have $f=0$ regardless of the extent to which parents are inbred, but $f$ of selfed progeny is contingent on the inbreeding coefficient of parents. As a consequence, two studies of the same population will yield different estimates of $\delta$ when one uses outbred parents and the other inbred parents.

The IDG program characterizes population-level ID with $\beta$, the rate that fitness declines as a function of the inbreeding coefficient (Charlesworth and Charlesworth, 1987). With outbred parents, $\beta$ is twice the difference in means between selfed and outcrossed progeny. If parents are fully inbred, $\beta$ is the simple difference. Variation among family means (outbred or inbred) is also contingent on both the experimental design and the genetic architecture of traits. IDG extracts estimates for genetic variance components $\left(V_{\mathrm{a}}, V_{\mathrm{d}}, C_{\mathrm{ad}}, V_{\mathrm{di}}\right)$ that depend only on the genetic architecture. These components are then used to calculate $\boldsymbol{I}_{1}$ and $\boldsymbol{I}_{2}$.

The accuracy of estimates for fixed effects ( $\beta$ and $M$ ), variance components (eg, $V_{\mathrm{a}}$ and $\left.V_{\mathrm{di}}\right)$, and derived statistics $\left(\boldsymbol{I}_{\mathbf{1}}\right.$ and $\left.\boldsymbol{I}_{\mathbf{2}}\right)$ depends on the experimental design, on sample sizes, and on the true values for parameters. The primary advantage of Design 1 is simplicity. A full experiment can be concluded in two generations with parents grown from field collected seed (eg, Kalisz, 1989). The design also provides an elegant means to estimate population-level ID, as each family provides 'paired estimates' of outbred and inbred trait values (the means from each subfamily in Figure 1a). Accounting for this pairing increases estimation accuracy when inbred and outbred means are positively correlated across families (Johnston and Schoen, 1994).

We distinguish four cases of Design 1 based on the level of parental inbreeding and the number of sires per dam. Some guidance regarding the relative accuracy of estimates for $V_{\mathrm{GI}}$ (and thus $\boldsymbol{I}_{\mathbf{1}}$ and $\boldsymbol{I}_{2}$ ) from these cases can be gained by noting the coefficients in Equations (4ad). All else equal, the $\mathrm{SE}$ for $V_{\mathrm{GI}}$ should be roughly proportional to the coefficients on the RHSs of these equations. Inspecting Equations $(4 a-d)$, it is clear that the $\mathrm{SE}$ for $V_{\mathrm{GI}}$ should be smaller with a single sire per dam than with many. The SE should be much smaller from experiments using fully inbred parents instead of outbred parents. This is not surprising given that selfed progeny of fully inbred parents are essentially inbred lines and thus provide a direct estimate for $V_{\mathrm{GI}}$.

A potential difficulty with Design 1 is that maternal effects cannot be distinguished from genetic effects in determining the resemblance among relatives. The reciprocal cross of Design 2 and the half-sib comparison of Design 3 allow the contribution of maternal effects to be estimated. The greater range of comparisons within Design 3 allows a more detailed genetic dissection of variation. In many studies, the large SEs for variance component estimates may prevent unambiguous conclusions. Such estimates may nonetheless prove valuable in a comparative context (eg, Husband and Schemske,
1996). A synthesis of results from different studies may tell us whether $I_{1}$ and $I_{2}$ are typically greater for morphological traits than life-history traits. Are $\boldsymbol{I}_{\mathbf{1}}$ and $I_{2}$ related to the mating system of a species? In short, the various questions typically asked about the magnitude of population-level ID could also be addressed to other aspects of genetic variation in fitness.

\section{Acknowledgements}

Norm Slade, Liza Holeski, and two anonymous reviewers provided useful comments on a preliminary draft of this paper. We gratefully acknowledge the support of NIH grant R01 GM60792-01A1, NSF grant DEB-0543052, and NSF Epscor.

\section{References}

Agren J, Schemske DW (1993). Outcrossing rate and inbreeding depression in two annual monoecious herbs, Begonia hirsute a and b. Semiovata Evol 47: 125-135.

Barrett SCH, Charlesworth D (1991). Effects of a change in the level of inbreeding on the genetic load. Nature 352: 522-524.

Byers DL, Waller DM (1999). Do plant populations purge their genetic load? Effects of population size and mating history on inbreeding depression. Ann Rev Ecol Systemat 30: 479-513.

Campbell RB (1986). The interdependence of mating structure and inbreeding depression. Theor Pop Biol 30: 232-244.

Carr DE, Fenster CB, Dudash MR (1997). The relationship between mating-system characters and inbreeding depression in Mimulus guttatus. Evolution 51: 363-372.

Chang S-M, Rausher MD (1999). The role of inbreeding depression in maintaining the mixed mating system of the common morning glory, Ipomea purpurea. Evolution 53: 1366-1376.

Charlesworth B, Hughes KA (2000). The maintenance of genetic variation in life history traits. In: Singh RS, Krimbas CB (eds) Evolutionary Genetics from Molecules to Morphology. Cambridge University Press: Cambridge, UK. pp 369-392.

Charlesworth D, Charlesworth B (1987). Inbreeding depression and its evolutionary consequences. Ann Rev Ecol Systemat 18: 237-268.

Charlesworth D, Morgan MT, Charlesworth B (1990). Inbreeding depression, genetic load, and the evolution of outcrossing rates in a multilocus system with no linkage. Evolution 44: $1469-1489$.

Cockerham CC (1983). Covariances of relatives from selffertilization. Crop Sci 23: 1177-1180.

Cockerham CC, Weir BS (1984). Covariances of relatives stemming from a population undergoing mixed self and random mating. Biometrics 40: 157-164.

Crow JF (1987). Muller, dobzhansky, and overdominance. J Hist Biol 20: 351-380.

Darwin CR (1876). The Effects of Cross- and Self-Fertilisation in the Vegetable Kingdom. John Murray: London.

Falconer DS, Mackay TFC (1996). Introduction to Quantitative Genetics. Prentice Hall: London.

Feller W (1968). An Introduction to Probability Theory and its Applications. John Wiley and sons: New York.

Fishman L (2001). Inbreeding depression in two populations of Arenaria uniflora (caryophyllaceae) with contrasting mating systems. Heredity 86: 184-194.

Fox CW (2005). Problems in measuring among-family variation in inbreeding depression. American J Botany 92: 1929-1932.

Harris DL (1964). Genotypic covariances between inbred relatives. Genetics 50: 1319-1348.

Holsinger KE (1988). Inbreeding depression doesn't matter: the genetic basis of mating-system evolution. Evolution 42: 1235-1244.

Hughes KA (1997). Quantitative genetics of sperm precedence in Drosophila melanogaster. Genetics 145: 139-151. 
Husband BC, Schemske DW (1996). Evolution of the magnitude and timing of inbreeding depression in plants. Evolution 50: $54-70$.

Johnston MO, Schoen DJ (1994). On the measurement of inbreeding depression. Evolution 48: 1735-1741.

Kalisz S (1989). Fitness consequences of mating system, seed weight, and emergence date in a winter annual, Colinsia verna. Evolution 43: 1263-1272.

Keller LF, Waller DM (2002). Inbreeding effects in wild populations. Trend Ecol Evol 17: 230-241.

Kelly JK (1999). An experimental method for evaluating the contribution of deleterious mutations to quantitative trait variation. Genetic Research 73: 263-273.

Kelly JK (2003). Deleterious mutations and the genetic variance of male fitness components in Mimulus guttatus. Genetics 164: $1071-1085$

Kelly JK (2005a). Epistasis in monkeyflowers. Genetics 171: 1917-1931.

Kelly JK (2005b). Family level inbreeding depression and the evolution of plant mating systems. New Phytolo 165: 55-62.

Kelly JK, Arathi HS (2003). Inbreeding and the genetic variance of floral traits in Mimulus guttatus. Heredity 90: 77-83.

Koelewijn HP, Koski V, Savolainen O (1999). Magnitude and timing of inbreeding depression in scots pine (Pinus sylvestris L.). Evolution 53: 758-768.

Lande R, Schemske DW (1985). The evolution of self-fertilization and inbreeding depression in plants. I. Genetic models. Evolution 39: 24-40.

Lynch M, Conery J, Burger R (1995). Mutation accumulation and the extinction of small populations. Am Nat 146: 489-518.

Lynch M, Walsh B (1998). Genetics and Analysis of Quantitative Characters. Sinauer Associates: Sunderland, MA.

Maynard Smith J (1978). The Evolution of Sex. Cambridge University Press: Cambridge.

Moorad JA, Wade MJ (2005). A genetic interpretation of the variation in inbreeding depression. Genetics 170: 1373-1384.

Mutikainen P, Delph LF (1998). Inbreeding depression in Gynodioecious lobelia siphilitica: Among-family differences override between-morph differences. Evolution 52: 1572-1582.

Rao G-Y, Widen B, Andersson S (2002). Patterns of inbreeding depression in a population of Brassica cretica (Brassicaceae): evidence from family-level analyses. Biol J Linn Soc 76: 317-325.

Rice JA (1989). Mathematical Statistics and Data Analysis. Wadsworth and Brooks: Pacific Grove, California.

Robertson A (1952). The effect of inbreeding on the variation due to recessive genes. Genetics 37: 189-207.

Shaw RG, Byers DL, Shaw FH (1998). Genetic components of variation in Nemophila menziesii undergoing inbreeding: morphology and flowering time. Genetics 150: 1649-1661.

Shultz S, Willis J (1995). Individual variation in inbreeding depression - the roles of inbreeding history and mutation. Genetics 141: 1209-1223.

Simmons MJ, Crow JF (1977). Mutations affecting fitness in Drosophila populations. Ann Rev Genet 11: 49-78.

Stone JL, Motten AF (2002). Anther-stigma separation is associated with inbreeding depression in Datura stramonium, a predominantly self-fertilizing annual. Evolution 56: 2187-2195

Takano T, Kusakabe S, Mukai T (1987). The genetic structure of natural populations of Drosophila melanogaster. Xx. Comparisons of genotype environment interaction in viability between a northern and a southern population. Genetics 117: 245-254.

Takebayashi N, Delph LF (2000). An association between a floral trait and inbreeding depression. Evolution 54: 840-846.

Uyenoyama MK, Holsinger KE, Waller DM (1993). Ecological and genetic factors directing the evolution of self-fertilization. Oxford Survey Evol Biol 9: 327-381.

Uyenoyama MK, Waller DM (1991). Coevolution of selffertilization and inbreeding depression. Iii. Homozygous lethal mutations at multiple loci. Theor Pop Biol 40: 173-210.
Vogler DW, Filmore K, Stephenson AG (1999). Inbreeding depression in campanula rapunculoides l. I. A comparison of inbreeding depression in plants derived from strong and weak self-incompatibility phenotypes. J Evol Biol 12: 483-494.

Willis JH (1993). Partial self fertilization and inbreeding depression in two populations of Mimulus guttatus. Heredity 71: $145-154$.

Willis JH (1999a). Inbreeding load, average dominance, and the mutation rate for mildly deleterious alleles in Mimulus guttatus. Genetics 153: 1885-1898.

Willis JH (1999b). The role of genes of large effect on inbreeding depression in Mimulus guttatus. Evolution 53: 1678-1691.

Wright S (1952). The genetics of quantitative variability. In: Reeve ECR, Waddington $\mathrm{CH}$ (eds) Quantitative Inheritance. Her Majesty's Stationary Office: London, UK. pp 5-41.

\section{Appendix: Algebraic relationships between observational and causal variance components}

Each comparisons among relatives depicted in Figure 1 is expressed as functions of genetic variance components in Table 2 of Cockerham and Weir (1984). We assume that the parents are either outbred $(f=0)$ or fully inbred $(f=1)$ and that there are only two alleles at each polymorphic QTL. Thus, the observational components $\left(V_{\mathrm{O}}, V_{\mathrm{I}}, C_{\mathrm{HS}}, C_{\mathrm{OO}}, C_{\mathrm{OS}}, C_{\mathrm{SS}}\right)$ can be expressed as a function of four genetic components $\left(V_{\mathrm{a}}, C_{\mathrm{ad}}, V_{\mathrm{d}}\right.$, and $\left.V_{\mathrm{di}}\right)$ and one or two environmental components $\left(V_{\mathrm{E}}\right.$ and possibly $V_{\mathrm{M}}$ ). For Designs 1 and 2, causal component estimates are extracted from estimates of the observational components using the formulas given below.

\section{Design 1}

For Case 1 in which parents are outbred and there is a single sire per dam:

$$
\begin{aligned}
V_{\mathrm{E}} & =\left(-7 C_{\mathrm{OO}}+8 C_{\mathrm{OS}}-4 C_{\mathrm{SS}}+V_{\mathrm{I}}+2 V_{\mathrm{O}}\right) / 3 \\
V_{\mathrm{a}} & =\left(5 C_{\mathrm{OO}}+8 C_{\mathrm{OS}}-4 C_{\mathrm{SS}}+V_{\mathrm{I}}-V_{\mathrm{O}}\right) / 3 \\
V_{\mathrm{d}} & =2\left(C_{\mathrm{OO}}-8 C_{\mathrm{OS}}+4 C_{\mathrm{SS}}-V_{\mathrm{I}}+V_{\mathrm{O}}\right) / 3 \\
C_{\mathrm{ad}} & =-2\left(5 C_{\mathrm{OO}}+2 C_{\mathrm{OS}}-4 C_{\mathrm{SS}}+V_{\mathrm{I}}-V_{\mathrm{O}}\right) / 3 \\
V_{\mathrm{di}} & =4\left(3 C_{\mathrm{OO}}-2 C_{\mathrm{SS}}+V_{\mathrm{I}}-V_{\mathrm{O}}\right) \\
\text { and } & V_{\mathrm{GI}}=2\left(C_{\mathrm{OO}}+V_{\mathrm{I}}-V_{\mathrm{O}}\right)
\end{aligned}
$$

For Case 2 where parents are outbred and there are many sires per dam:

$$
\begin{aligned}
V_{\mathrm{E}} & =\left(-28 C_{\mathrm{OO}}+32 C_{\mathrm{OS}}-16 C_{\mathrm{SS}}+4 V_{\mathrm{I}}+V_{\mathrm{O}}\right) / 5 \\
V_{\mathrm{a}} & =4 C_{\mathrm{OO}} \\
V_{\mathrm{d}} & =4\left(2 C_{\mathrm{OO}}-8 C_{\mathrm{OS}}+4 C_{\mathrm{SS}}-V_{\mathrm{I}}+V_{\mathrm{O}}\right) / 5 \\
C_{\mathrm{ad}} & =-4\left(2 C_{\mathrm{OO}}-C_{\mathrm{OS}}\right) \\
V_{\mathrm{di}} & =8\left(18 C_{\mathrm{OO}}-12 C_{\mathrm{OS}}+C_{\mathrm{SS}}+V_{\mathrm{I}}-V_{\mathrm{O}}\right) / 5 \\
\text { and } & V_{\mathrm{GI}}=8\left(3 C_{\mathrm{OO}}-2 C_{\mathrm{OS}}+C_{\mathrm{SS}}+V_{\mathrm{I}}-V_{\mathrm{O}}\right) / 5
\end{aligned}
$$


For Case 3 with fully inbred parents and a single sire per dam, it is not possible to distinguish genetic variance components, but

$$
V_{\mathrm{GI}}=\left(C_{\mathrm{OO}}+3 C_{\mathrm{SS}}+V_{\mathrm{I}}-V_{\mathrm{O}}\right) / 4
$$

Finally, when parents are fully inbred and there are many sires per dam (Case 4):

$$
\begin{aligned}
& V_{\mathrm{E}}=V_{\mathrm{I}}-C_{\mathrm{SS}} \\
& V_{\mathrm{a}}=2 C_{\mathrm{OO}} \\
& V_{\mathrm{d}}=-2 C_{\mathrm{OO}}+C_{\mathrm{SS}}-V_{\mathrm{I}}+V_{\mathrm{O}} \\
& C_{\mathrm{ad}}=-2 C_{\mathrm{OO}}+C_{\mathrm{OS}} \\
& V_{\mathrm{di}}=4 C_{\mathrm{OO}}-4 C_{\mathrm{OS}}+C_{\mathrm{SS}}
\end{aligned}
$$

and

$$
V_{\mathrm{GI}}=C_{\mathrm{SS}}
$$

Essentially the same comparisons among relatives are contained within Design 2. However, the reciprocal crossing of parents allows $V_{\mathrm{M}}$ to be estimated. Also, because there is a single sire per dam, only cases 1 and 3 from above apply here. When there is no replication within sub-families and parents are outbred (eg, Willis, 1993), we cannot estimate $V_{\mathrm{GI}}$ (or $\boldsymbol{I}_{\mathbf{1}}$ and $\boldsymbol{I}_{\mathbf{2}}$ ). If parents are fully inbred however,

$$
V_{\mathrm{E}}=V_{\mathrm{O}}-C_{\mathrm{OO}}-V_{\mathrm{M}}
$$

and

$$
V_{\mathrm{GI}}=C_{\mathrm{OO}}+V_{\mathrm{I}}-V_{\mathrm{O}}
$$

